\title{
Anti-N-Methyl-D-Aspartate Receptor Encephalitis in a Young Woman with Ovarian Teratoma
}

\section{Giulia Magoga ${ }^{*}$, Irene Pascoli², Giuseppa Corrao², Enrico Busato ${ }^{2}$ and Antonella De Virgilio ${ }^{3}$}

${ }^{1}$ Department of Medical, Surgical and Health Sciences, University of Trieste, Trieste, Italy

${ }^{2}$ Department of Obstetrics and Gynecology, Santa Maria di Ca' Foncello Hospital, Treviso, Italy

${ }^{3}$ Department of Women's and Children's Health SDB Padova, Italy

*Corresponding author: Giulia Magoga, Department of Medical, Surgical and Health Sciences, University of Trieste, IRCCS Burlo Garofolo, 34137 Dell'Istria Street 65, Italy, Tel: +393478932740, E-mail: magogagiulia@gmail.com

Received: July 05, 2018; Accepted: July 26, 2018; Published: July 28, 2018

Citation: Magoga G, Pascoli I, Corrao G, Busato E, De Virgilio A (2018) Anti-N-Methyl-D-Aspartate Receptor Encephalitis in a Young Woman with Ovarian Teratoma. Vol.4 No.3:79.

\section{Abstract}

Background: Anti-N-Methyl-D-Aspartate (NMDA) Receptor encephalitis is a common cause of autoimmune encephalitis, usually associated with ovarian teratoma, especially in young women. It's important to recognize quickly this type of encephalitis in order to give the patient the better chance to recover.

Case: We report a typical case of anti-NMDA encephalitis associated to ovarian dermoid in a young woman. Neurological assessment revealed the presence of antiNMDA autoantibodies in cerebrospinal fluid and TC abdomen revealed the presence of an ovarian dermoid. Surgery to remove the mass was performed and immunotherapy was started. Now she's doing well, and she only has a little memory deficit.

Conclusion: Ovarian dermoid is common, instead enchephatis is a rare entity, so further investigations is needed to know more about this specific type of ovarian dermoid.

Keywords: Anti-N-Methyl-D-Aspartate; Autoimmune encephalitis; Psychiatric disorder; Electroencephalogram; Benign tumours

\section{Key Points}

1. Clinicians should always think about autoimmune encephalitis in case of psychiatric disorder especially in young women.

2. It is very important to recognize the disease as soon as possible in order to start therapy and to give the patient the better chance to achieve a completely recovery.

3. In our opinion gynecologist don't have to change their management in case of small ovarian dermoid because it is a very common ovarian benign neoplasm, whereas encephalitis is a rare condition.

\section{Introduction}

Symptoms of anti-N-Methyl-D-Aspartate (NMDA) Receptor encephalitis were described for the first time by Vitalini et al. in 2005 [1]. They described four cases of psychiatric disorders, memory loss and a decrease of level of consciousness associated with ovarian teratoma [1].

Now-a-days, it is widely known that Anti NMDA Encephalitis is the most common cause of autoimmune encephalitis after acute demyelinating encephalomyelitis [2]. The autoantibody responsible for the syndrome was described in 2007.

The NMDA Receptor is localized in post-synaptic membranes, it is a tetrameric complex composed by two subunits, NR1 and NR2. NR1 is the glycine-binding subunits, whereas NR2 is the glutamate-binding subunits; autoantibodies responsible for this type of encephalitis, specifically bind to an extracellular domain of the NR1 subunits [3].

Anti-NMDA encephalitis is usually associated with the presence of a neoplasm, mostly ovarian dermoid in young women [2]. We report a case of anti-NMDA encephalitis in a woman of 23 years associated with mature cystic teratoma, that is the most common type of ovarian germ cell tumours in young women between 20 and 30 years old [4].

\section{Case Presentation}

A 23-year-old woman was referred in March 2017 to the emergency department in "Ca' Foncello" Hospital in Treviso because of a headache. She had no past medical history and there was not history of substance abuse. Neurological assessment was negative, so she was discharged after being administrated pain relief medication.

After a few hours the patient came back to the hospital, accompanied by her parents, complaining of symptoms of an acute episode of psychotic behaviour. Basic blood evaluation 
was normal and cranial computer tomography was unremarkable, so she was admitted to the psychiatric department because clinicians suspected a psychiatric disorder, since a neurological examination was negative too. Electroencephalogram revealed a diffuse encephalitic insufficiency and over the next few days, she became unresponsive and developed catatonia.

After a few days the patient became progressively deeper in unconsciousness and for this reason diazepam and aloperidolo were prescribed. The patient quickly deteriorated and required intubation and intensive care monitoring; she also developed fever.

Neurological examination was performed again, and clinicians suspected the presence of autoimmune encephalitis, in particular, the anti-NMDAR type. For this reason, she underwent cerebrospinal fluid that was negative for infections and for other autoantibodies but showed high titles of antiNMDAR antibodies. So, she was admitted to the Neurological Department and underwent therapy with $1 \mathrm{~g} /$ day intravenous methylprednisolone for 5 days and then oral Prednisolone 50 $\mathrm{mg} /$ day combined with Intravenous Immunoglobulin $(0.4$ $\mathrm{g} / \mathrm{Kg}$ ) for 5 days.

Since, anti-NMDA encephalitis is usually caused by the presence of a dermoid cyst in young women [2], the patient underwent an abdominal computer tomography that showed the presence of a left ovarian dermoid cyst (Figure 1), confirmed by pelvic ultrasound.

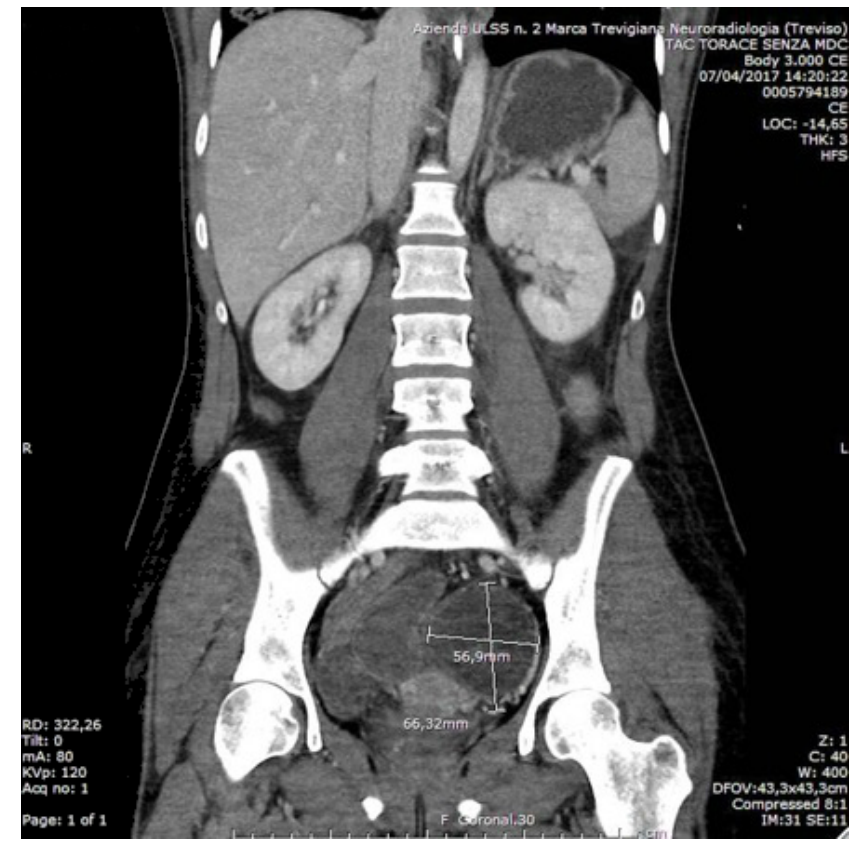

Figure 1 Abdominal CT revealing ovarian left cyst, $56.9 \times$ $66.3 \mathrm{~mm}$.

Then she underwent laparoscopy to remove the left cyst. During the surgery the cyst broke and some clear fluid leaked into the abdomen (Figure 2).

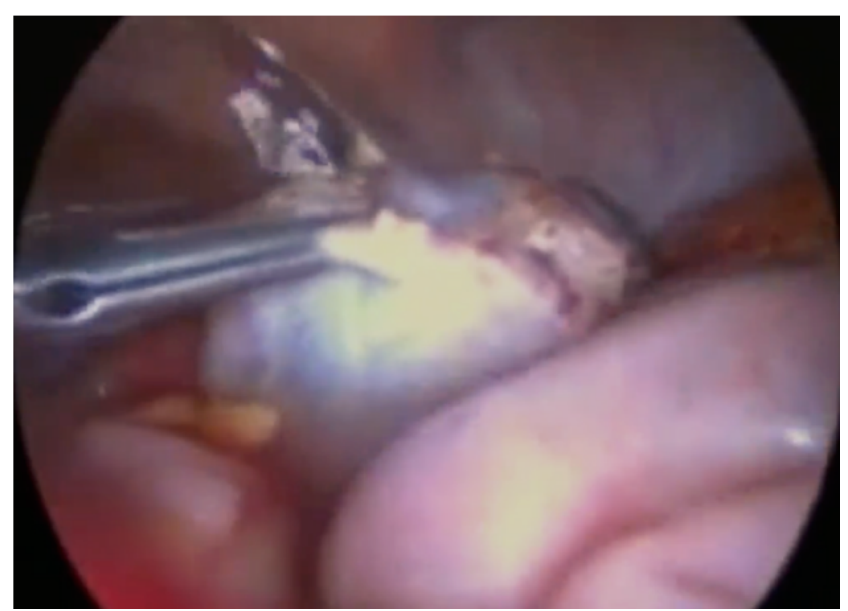

Figure 2 In this picture we can see the rupture of the left ovarian cyst with spillage of clear fluid.

The cyst didn't have any characteristic features typical of a dermoid cyst, as a matter of fact, in dermoid cysts there is usually hair and a lot of mucus and not clear fluid. However, histology revealed that the cyst was an ovarian teratoma and right ovarian biopsy was negative. The patient didn't know she had had a dermoid cyst before; moreover, she had a transvaginal ultrasound in December 2016 that revealed only the presence of a functional cyst on the left ovary.

The cyst was unilocular, $20 \times 14 \mathrm{~mm}$, an echoich, with regular internal and external surface, not vascularized at colour Doppler, and this is not a common ultrasound pattern for dermoid cyst [5]. After surgery she was doing well, but because the recovery was slow, she underwent second line therapy with Rituximab and Cyclophosphamide. She was discharged after about two months and now she's undergoing neurological follow up. After three months she had some problems with short-term memory and some involuntary movements on the right part of the mouth and right arm. Whereas after four months, when she came to neurological follow-up for the third administration of Cyclophosphamide, she was doing well, and the memory deficit was almost completely resolved.

\section{Discussion}

Mature cystic teratoma is the one of the most common benign ovarian neoplasm and is mostly common in young women [4]. They are benign tumours and patients are usually asymptomatic. Complications are rare, like torsion mostly common in big masses, and rupture with spillage of sebaceous material into the abdomen [6]. A rare condition associated with dermoid cyst is anti-NMDA encephalitis that is caused by the presence of nervous tissue into the cyst and by the production of autoantibodies against NMDA receptor [3].

In this manuscript we present a characteristic case of anti NMDA encephalitis associated with ovarian teratoma in a white woman, aged 23. NMDA Receptor is located in postsynaptic membranes and is very important for synaptic 
transmissions. It's composed by two subunits, NR1 and NR2, that bind respectively glycine and glutamate. Autoantibodies causing encephalitis are able to bind an extracellular domain of the NR1 subunits [3]. Symptoms are usually changes in behaviour, memory deficit, speech disorder, loss of consciousness, movement disorder and seizures [2], moreover about $70 \%$ of anti-NMDA encephalitis starts with prodromal symptoms like headache, fever, gastrointestinal disorders or upper respiratory-tract symptoms [7]. As a matter of fact, our patient referred to the emergency department complaining of a headache and only after a few hours symptoms worsened with changes in behaviour and loss of consciousness.

The diagnosis is possible after spinal tap and searching for anti-NDMA autoantibodies in cerebrospinal fluid [7]. If antiNMDA encephalitis is confirmed, it's recommended to perform MRI, computed tomography or pelvic ultrasound to find a neoplasm, responsible for the production of autoantibodies [7]. In particular, an ovarian dermoid is present between $31 \%$ and $50 \%$ of women affected by anti-NMDA encephalitis, aged 18 or more [3]. Treatment in presence of a neoplasm, consists of removal of the mass and first-line of immunotherapy, that is corticosteroids, intravenous immunoglobulins or plasma exchange [7].

In patients without tumour or in cases of persistence of symptoms, clinicians may use second line immunotherapy with Rituximab and Cyclophosphamide [7]. Our patient was very young and wasn't aware of having a dermoid cyst. Unfortunately, like other patients and considering the initial symptoms, she was referred to the psychiatric department and this could have delayed treatment. After removal of the ovarian mass, the girl began to recover but after first line immunotherapy, clinicians started the second line too, because of persistent neurological problems.

After the third administration of Cyclophosphamide the patient is doing well, and the recovery is almost complete; she has some memory deficit, but we are optimistic for future resolving of problems, since in literature it is reported a complete improvement in $81 \%$ of patients after removal of the tumour and immunotherapy after 24 months [7].

\section{Conclusion}

We have described a characteristic case of anti-NDMA encephalitis, associated with ovarian dermoid in a young woman. Now-a-days, it is known that Anti-NMDA encephalitis is the most common cause of autoimmune encephalitis after acute demyelinating encephalomyelitis [2], and mature cystic teratoma is one of the most common benign ovarian neoplasm. For this reason, it is important to know this specific type of autoimmune encephalitis and to start the right therapy to improve outcomes.

Recognizing the disease as soon as possible is very important to give the patient the best chance to achieve a completely recovery. Ovarian dermoid can be associated to anti-NMDA encephalitis, usually in young women and so, clinicians have to search for a tumour after diagnosis of this type of encephalitis, to remove it. However, we think that we don't have to change our management with ovarian dermoid, since they are benign tumours and anti-NMDA encephalitis is a rare entity. Moreover, to our knowledge, there's no case of anti-NMDA encephalitis in women who knew to have an ovarian cyst; in literature there are only cases of anti-NMDA encephalitis associated to ovarian dermoid, that the patient wasn't aware of having. So, it's reasonable thinking that ovarian dermoid is not a risk factor for encephalitis, but it depends on which type of tissue is expressed into the cyst. If there is nervous tissue expressing autoantibodies, encephalitis develops very quickly, whereas, if there isn't production of auto-antibodies, ovarian dermoid behaves like a benign mass, without giving any symptoms.

Unfortunately, we don't have a record of pelvic ultrasound performed before surgery because the patient underwent ultrasound during her stay in intensive care, but it would be interesting to study echography features of the cyst. As a matter of fact, during surgery the cyst didn't appear like a typical dermoid, since the fluid was clear and there wasn't mucus or hair. It may be the case in ultrasound that this specific type of dermoid expressing nervous tissue, has a typical appearance, that is not common to teratoma. To be able to diagnose dermoid with nervous tissue could be helpful to predict the risk of developing anti-NMDA encephalitis and this could be a matter for further study.

\section{References}

1. Vitaliani R, Mason W, Ances B, Zwerdling T, Jiang Z, et al. (2005) Paraneoplastic encephalitis, psychiatric symptoms, and hypoventilation in ovarian teratoma. Ann Neurol 58: 594-604.

2. Titulaer MJ, McCracken L, Gabilondo I, Armangué T, Glaser C, et al. (2013) Treatment and prognostic factors for long-term outcome in patients with anti-NMDA receptor encephalitis: an observational cohort study. Lancet Neurol 12: 157-165.

3. Dalmau J, Gleichman AJ, Hughes EG, Rossi JE, Peng X, et al. (2008) Anti-NMDA-receptor encephalitis: A case series and analysis of the effects of antibodies. Lancet Neurol 7: 1091-1098.

4. Ayhan A, Bukulmez O, Genc C, Karamursel BS, Ayhan A, et al. (2000) Mature cystic teratomas of the ovary: case series from one institution over 34 years. Eur J Obstet Gynecol Reprod Biol 88: 153-157.

5. Cohen L, Sabbagha R (1993) Echo patterns of benign cystic teratomas by transvaginal ultrasound. Ultrasound Obstet Gynecol 3: 120-123.

6. Deffieux X, Thubert T, Huchon C, Demoulin G, Rivain AL, et al. (2013) Complications des tumeurs ovariennes présumées bénignes. Journal de Gynécologie Obstétrique et Biologie de la Reproduction 42: 816-832.

7. Dalmau J, Lancaster E, Martinez Hernandez E, Rosenfeld MR, Balice-Gordon R (2011) Clinical experience and laboratory investigations in patients with anti-NMDAR encephalitis. Lancet Neurol 10: 63-74. 